\title{
Relation between high-frequency properties and direction of anisotropy magnetic field in magnetic thin film for RF inductor applications
}

\author{
Makoto Sonehara, Member, IEEE, and Toshiro Sato, Member, IEEE
}

Spin Device Technology Center, Shinshu University, 4-17-1 Wakasato, Nagano 380-8553, Japan

\begin{abstract}
A relation between high-frequency properties, and angle $\theta$ of the easy axis in soft magnetic film and ac applied field was investigated in order to fabricate the film for RF spiral inductor without a localized EMI (Electro-Magnetic Interference). This relationship was measured using a soft $\mathrm{CoFeSiO} / \mathrm{SiO}_{2}$ granular multilayer magnetic thin film and simulated by using LLG (LandauLifshitz-Gilbert) micromagnetic simulator. The measured and calculated frequency dependence of the film's complex relative permeability, $\mu_{\mathrm{r}}$ ' and $\mu_{\mathrm{r}}$ ", compared well with $10 \%$ mean error. The real part of complex relative permeability $\mu_{\mathrm{r}}$ ' was dependent on approximately $\sin ^{2} \theta$. The ferromagnetic resonance (FMR) frequency, $f_{\mathrm{r}}$, and the FMR half-width, $\Delta f_{\mathrm{r}}$ were not dependent on the angle, $\theta$. They were estimated to be $2.54 \mathrm{GHz}$ and $0.3 \mathrm{GHz}$, respectively. These results are significant for the design of various magnetic thin film spiral inductors in RF integrated circuits without localized EMI.
\end{abstract}

Index Terms - Magnetic thin film spiral inductor, LLG micromagnetic simulator, Direction of anisotropy magnetic field, $\mathrm{CoFeSiO} / \mathrm{SiO}_{2}$ granular multilayer magnetic film, Permeability, FMR frequency.

\section{INTRODUCTION}

$\mathrm{M}$ iniature Radio Frequency (RF) inductors with magnetic thin films are currently under research for use in impedance matching networks and lumped element filters for applications including cellular phones, laptop computers, and various RF communication devices [1]-[4]. Most RF inductors have a spiral and planar design because their inductance densities are typically higher than the densities of meander designs. This is primarily due to the larger positive mutual inductances, therefore, making them suitable for RF integrated circuits. The inductance $L$ and the resistivity $R$ in the RF spiral inductors were affected by the complex permeability $\mu$ ' and $\mu$ ” in the magnetic thin films, especially. Generally, the magnetic films with uniaxial anisotropic magnetic field in the RF inductors were established such as Fig. 1 [1]-[4]. In this case, an inductance of the area A in Fig. 1 (a) was enhanced by high permeability in the magnetic thin film. On the other hand, the inductance of the area $\mathrm{B}$ was equal to that of the air core case, because the permeability $\mu^{\prime}=\mu_{0}$ in the magnetic thin film. In addition, it was impossible to suppress cross-talk noise in the area B, so then localized EMI was occurred.

Hence, an RF inductor with 45 degrees of the direction of high-frequency excitation and the hard axis was fabricated such as Fig. 1 (b). In this case, it was possible to obtain high inductance and suppress the cross-talk noise in all areas of the inductor, because all areas had high permeability $\mu$ '. However, it was difficult to estimate the complex permeability $\mu$ ' and $\mu$ " in the magnetic film by the LLG equation [5] and design the inductors.

Manuscript received Xxxxx XX, 2012; revised Xxxxx XX, 2012. Current version published Xxxxx XX, 2012. Corresponding author: Makoto Sonehara (e-mail: makoto@shinshu-u.ac.jp; optional phone: +81-26-269-5198; optional fax: +81-26-269-5215)

Digital Object Identifier 10.1109/TMAG.2012.xxxxxxx

In this work, the authors investigated a relation between high-frequency properties and the direction of the anisotropy magnetic field in the magnetic film by experiments and simulations by using LLG micromagnetic simulator.

\section{EXPERIMENT}

\section{A. Fabrication of magnetic thin film}

$\mathrm{CoFeSiO} / \mathrm{SiO}_{2}$ granular multilayer films with sharp ferromagnetic resonance (FMR) peak were selected for the
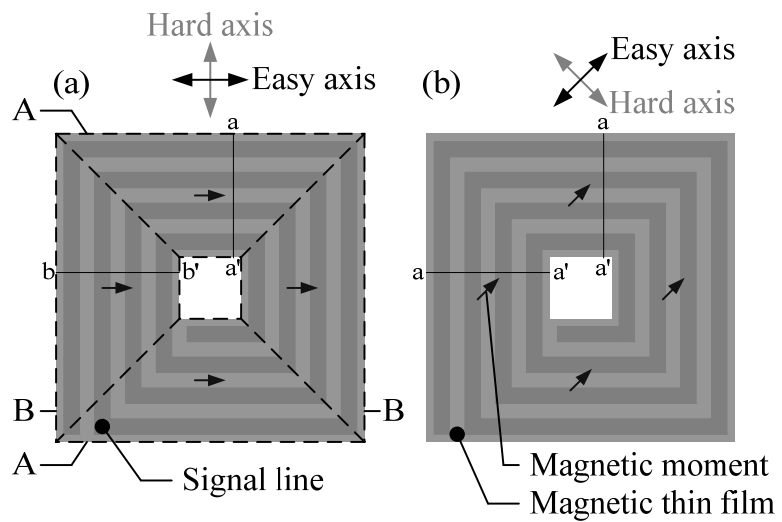

Fig. 1. Schematic of (a) conventional and (b) novel RF spiral planar inductors for RF circuits.

(a)

(b)

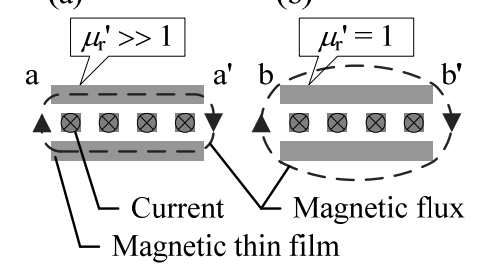

Fig. 2. Schematic of cross section (a) a-a' and (b) b-b' in the inductors in Figure 1. 
magnetic thin film [6]. They were deposited on a surfaceoxidized (100) silicon substrate at room temperature by cosputtering $\mathrm{Co}_{80} \mathrm{Fe}_{20}$ alloy and $\mathrm{SiO}_{2}$ targets using an inductively coupled RF sputtering system under a pressure of about $0.2 \mathrm{~Pa}$ of pure Ar gas. During deposition, a magnetic field of about 8 $\mathrm{kA} / \mathrm{m}$ was applied to the film plane in order to introduce inplane uniaxial magnetic anisotropy. The total thickness of the multilayer films was regulated to be about $50 \mathrm{~nm}$. The granular multilayer films were diced to $4 \mathrm{~mm}$ square size by using a dicer (Disco Corporation; DAD3220).

\section{B. Measurement}

The frequency dependence of permeability in the range from $1 \mathrm{MHz}$ to $9 \mathrm{GHz}$ was measured by using a high-frequency permeameter (Ryowa Electronics Co., Ltd.; PMM-9G1) with the sample size of $4 \mathrm{~mm}$ square size in the granular multilayer film [7].

\section{Simulation}

\section{A. Micromagnetic simulator}

The behavior of magnetic moment of the magnetic thin film was analyzed by using an LLG Micromagnetics Simulator (by M. R. Scheinfein) [8]. The behavior of magnetic moment was calculated on the basis of following basic equations.

$$
\begin{aligned}
\frac{\mathrm{d} \boldsymbol{M}}{\mathrm{d} t} & =-\frac{\gamma}{1+\alpha^{2}} \boldsymbol{M} \times \boldsymbol{H}_{\mathrm{eff}}-\frac{\gamma \alpha}{\left(1+\alpha^{2}\right) M_{\mathrm{s}}} \boldsymbol{M} \times\left(\boldsymbol{M} \times \boldsymbol{H}_{\mathrm{eff}}\right) \\
\boldsymbol{H}_{\mathrm{eff}} & =-\frac{\partial\left(E_{\mathrm{ku}}+E_{\mathrm{kc}}+E_{\mathrm{ks}}+E_{\mathrm{s}}+E_{\mathrm{h}}+E_{\mathrm{ex}}\right)}{\partial \boldsymbol{M}}
\end{aligned}
$$

where $\boldsymbol{M}$ is the magnetization, $M_{\mathrm{s}}$ is the saturation magnetization, $\gamma$ is the gyromagnetic constant, $\alpha$ is the damping constant, $\boldsymbol{H}_{\text {eff }}$ is the effective magnetic field, $E_{\mathrm{ku}}$ is the uniaxial magnetocrystalline anisotropy energy, $E_{\mathrm{kc}}$ is the cubic magnetocrystalline anisotropy energy, $E_{\mathrm{ks}}$ is the surface magnetocrystalline anisotropy energy, $E_{\mathrm{s}}$ is the selfmagnetostatic field energy, $E_{\mathrm{h}}$ is the external field energy, and $E_{\text {ex }}$ is the exchange energy.

\section{B. Simulation model}

In the LLG simulation, as shown in Fig. 3, each parameter was established according to the experimental results. A model for simulation with $x-y-z$ three dimensional coordinate composed of $1 \mathrm{~mm} \times 1 \mathrm{~mm}$ area in $x-y$ plane, and thickness $t_{\mathrm{F}}$ of $50 \mathrm{~nm}$ in the $z$-axis. The saturation magnetization $M_{\mathrm{s}}$ of $1.44 \mathrm{~T}$ and the uniaxial anisotropy field, $H_{\mathrm{k}}$, of $4.54 \mathrm{kA} / \mathrm{m}$ obtained in the magnetic film were given to the model. The direction of the uniaxial anisotropy field $H_{\mathrm{k}}$ was established in the $x-y$ plane. An angle $\theta$ of the direction and $y$-axis ranged from $0^{\circ}$ to $90^{\circ}$. Namely $y$-axis was the easy axis when $\theta=0^{\circ}$. On the other hand, $y$-axis was the hard axis when $\theta=90^{\circ}$. The ac field $h_{y}$ with amplitude of $8 \mathrm{~A} / \mathrm{m}$ was applied in the $y$-axis. The damping constant $\alpha$ of 0.007 was given to the model [7].

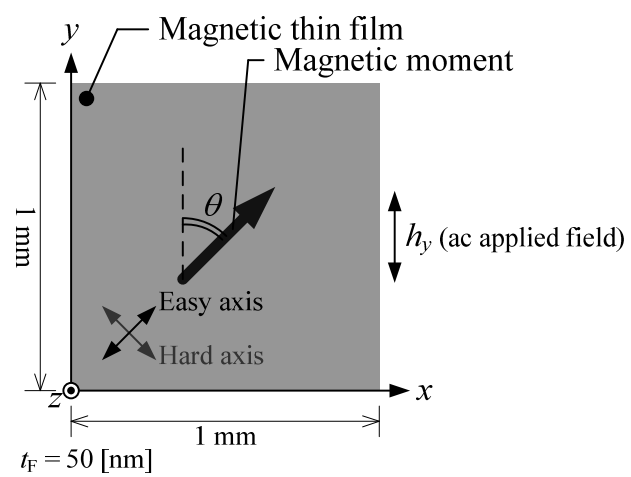

Fig. 3. Structure for LLG micromagnetic simulation of the $\mathrm{CoFeSiO} / \mathrm{SiO}_{2}$ granular multilayer film.

\section{RESULTS AND DISCUSSION}

\section{A. Frequency dependence of complex relative permeability}

Figure 4 shows the frequency dependence of the complex relative permeability and the quality factor, $Q$, which were measured and calculated for the $\mathrm{CoFeSiO} / \mathrm{SiO}_{2}$ granular multilayer films at $\theta=90^{\circ}, 60^{\circ}$, and $45^{\circ}$. The $Q$ is equivalent to $\mu_{\mathrm{r}}$ ' / $\mu_{\mathrm{r}}$ '. From Fig. 4, the calculated result agreed well with the experimental one. Their mean percent error was estimated about $10 \%$. However the experimental data in the vicinity of the FMR frequency $f_{\mathrm{r}}$ which is about $2.54 \mathrm{GHz}$ deviate from calculated one. The authors considered that the reason is a dispersion of the uniaxial anisotropy field in the films and a measurement error. However, the detailed reason is currently not clear. Both $f_{\mathrm{r}}$ and $\Delta f_{\mathrm{r}}$ were not dependent on $\theta$. The FMR half-width $\Delta f_{\mathrm{r}}$ was estimated about $0.3 \mathrm{GHz}$ from the results. The value is less than half that of generally ferromagnetic films for high-frequency devices [9], [10]. Therefore, the $\mathrm{CoFeSiO} / \mathrm{SiO}_{2}$ granular multilayer film is expected as the magnetic thin film for the RF with low loss, because the FMR loss is small around the FMR frequency.

\section{$B$. Relation between the angle $\theta$ and complex relative permeability}

Figure 5 shows the relation between the angle $\theta$, complex relative permeability $\mu_{\mathrm{r}}^{\prime}, \mu_{\mathrm{r}}^{\prime}$, and the quality factor $Q$ measured and calculated for the $\mathrm{CoFeSiO} / \mathrm{SiO}_{2}$ granular multilayer films at $1 \mathrm{GHz}$ (a), and $2 \mathrm{GHz}$ (b). The imaginary part of relative permeability $\mu_{\mathrm{r}}$ ” in Fig. 5 (a) was excluded, because the data had large measurement error. From Fig. 5, the experimental data are indeed consistent with calculated one approximately. The real part of the complex relative permeability $\mu_{\mathrm{r}}$ ' is described by the following equation;

$$
\mu_{\mathrm{r}}{ }^{\prime}=\left(\mu_{\mathrm{rh}}{ }^{\prime}-1\right) \sin ^{2} \theta+1
$$

where $\mu_{\mathrm{rh}}$ ' is the real part of complex relative permeability in the hard axis $\mu_{\mathrm{r}}$ '. The authors considered that this result agrees with following equation; 


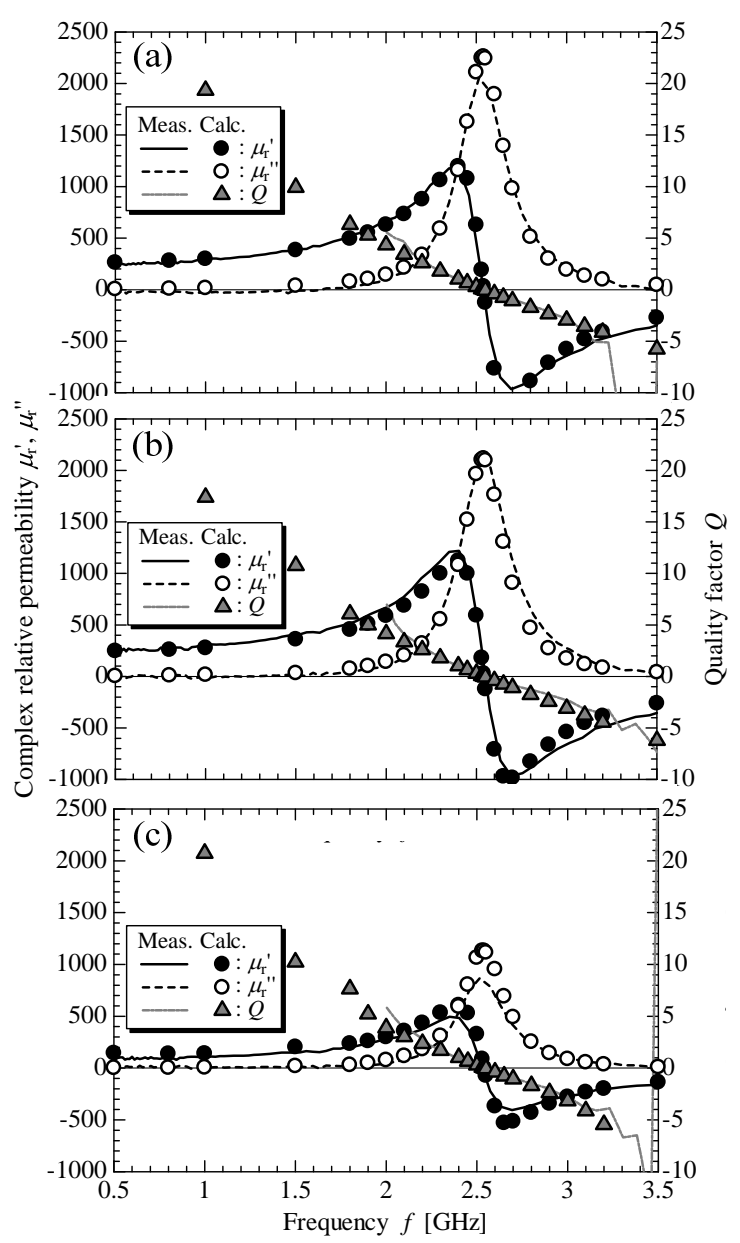

Fig. 4. Typical of frequency dependence of complex relative permeability and quality factor measured and calculated for the $\mathrm{CoFeSiO} / \mathrm{SiO}_{2}$ granular multilayer films, $\theta=90^{\circ}$ (a), $60^{\circ}$ (b), and $45^{\circ}$ (c).

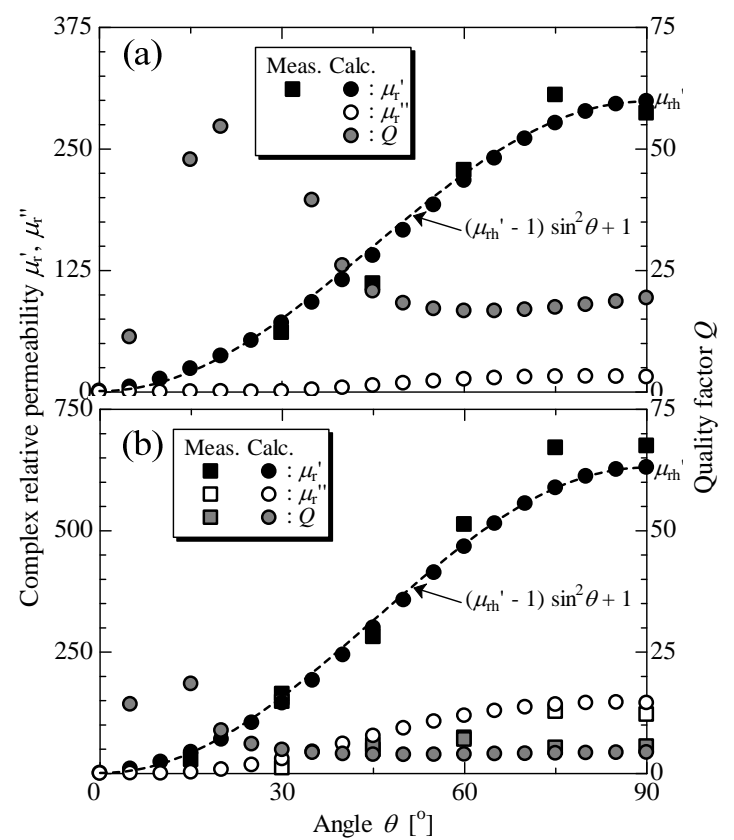

Fig. 5. Relation between the angle $\theta$, complex relative permeability $\mu_{r}^{\prime}, \mu_{r}^{\prime \prime}$, and quality factor $Q$ which were measured and calculated for the $\mathrm{CoFeSiO} / \mathrm{SiO}_{2}$ granular multilayer films at $1 \mathrm{GHz}(\mathrm{a})$, and $2 \mathrm{GHz}(\mathrm{b})$.

$$
E_{\mathrm{ku}}=K_{\mathrm{u}} \sin ^{2} \theta
$$

where $K_{\mathrm{u}}$ is the anisotropy constant. Hence the uniaxial magnetocrystalline anisotropy energy $E_{\mathrm{ku}}$ in the $\mathrm{CoFeSiO} / \mathrm{SiO}_{2}$ granular multilayer film is governed mainly.

The quality factor $Q$ is approximately constant in $\theta \geq 45^{\circ}$ in

Fig. 5. On the other hand, $Q$ has the peak in $\theta \cong 20^{\circ}$ in Fig. 5 . These reasons are currently not clear.

\section{CONCLUSIONS}

The relation between high-frequency properties and the direction of the anisotropy magnetic field in the $\mathrm{CoFeSiO} / \mathrm{SiO}_{2}$ granular multilayer magnetic thin films for RF inductor applications were measured and simulated using an LLG micromagnetic simulator. The results obtained are as follows:

(1) The measured and calculated frequency dependence of the complex relative permeability for the $\mathrm{CoFeSiO} / \mathrm{SiO}_{2}$ granular multilayer films compared well with a mean percent error of $10 \%$.

(2) The real part of complex relative permeability, $\mu_{\mathrm{r}}$ ', was approximately dependent on Eq. (3). This result was caused by the uniaxial magnetocrystalline anisotropy energy $E_{\mathrm{ku}}$ as Eq. (4).

(3) The FMR frequency and the FMR half-width were not dependent on the angle, $\theta$, of the direction of the easy axis and ac applied field. They were estimated to be $2.54 \mathrm{GHz}$ and $0.3 \mathrm{GHz}$, respectively.

These results are significant for the design of various magnetic thin film spiral inductors in the RF integrated circuits without the localized EMI.

\section{ACKNOWLEDGMENT}

The corresponding author really thank to Dr. Kenji Ikeda, R\&D Center, Taiyo Yuden Co., Ltd. for assisting the fabrication of $\mathrm{CoFeSiO} / \mathrm{SiO}_{2}$ granular multilayer films.

A part of this research was supported by "Adaptable and Seamless Technology Transfer Program (A-STEP; AS232Z01011B), Japan Science and Technology Agency”.

\section{REFERENCES}

[1] M. Yamaguchi, R. Baba, and K.-I. Arai, "Sandwich type ferromagnetic RF integrated inductor”, Microwave Symposium Digest, 2001 IEEE MTT-S International, Vol.1, pp.185-188 (2001).

[2] D. S. Gardner, G. Schrom, F. Paillet, B. Jamieson, T. Karnik, and S. Borkar, "Review of On-Chip Inductor Structures with Magnetic Films", IEEE Transactions on Magnetics, Vol.45, No.10, pp.4760-4766 (2009).

[3] K. Ikeda, T. Suzuki, T. Maruyama, T. Minemura, M. Sonehara, and T. Sato, "Double Spiral Inductors Using $\mathrm{CoFeSiO} / \mathrm{SiO}_{2}$ Multilayer Granular Films with Very Narrow Ferromagnetic Resonance Bandwidth”, J. Magn. Soc. Jpn., Vol.34, No.2, pp.123-130 (2010). (in Japanese)

[4] T. Maruyama, Y. Obinata, M. Sonehara, K. Ikeda, and Toshiro Sato, "Increase of Q-Factor of RF Magnetic Thin Film Inductor by Introducing Slit-Patterned Magnetic Thin Film and Multiline-Conductor Spiral Coil”, IEEE Transactions on Magnetics, Vol.47, No.10, pp. 31963199 (2011).

[5] Y. Shimada, J. Numazawa, Y. Yoneda, and A. Hosono, "Absolute Value Measurements of Thin Film Permeability”, J. Magn. Soc. Jpn., Vol.15, No.2, pp.327-330 (1991). (in Japanese) 
[6] K. Ikeda, T. Suzuki, and T. Sato, "CoFeSiO/SiO 2 Multilayer Granular Films With Very Narrow Ferromagnetic Resonant Linewidth”, IEEE Transactions on Magnetics, Vol.45, No.10, pp.4290-4293 (2009).

[7] M. Yamaguchi, Y. Miyazawa, K. Kaminishi, and K.-I. Arai, “A new 1 MHz-9 GHz thin film permeameter using a side-open TEM cell and a planar shielded-loop coil”, Trans. Magn. Soc. Jpn., Vol.3, No.4, pp.137140 (2003).

[8] M. Sonehara, T. Ishikawa, K. Inagaki, T. Sato, K. Yamasawa, Y. Miura and H. Fujiwara, "Ferromagnetic Resonance Simulation of Mn-Ir/Fe-Si Exchange-Coupled Film on Basis of LLG Equation Taking Exchange-
Stiffness Dispersion Model into Account”, J. Magn. Soc. Jpn., Vol.31, No.3, pp.153-158 (2007).

[9] M. Sonehara, T. Sugiyama, T. Sato, K. Yamasawa, and Y. Miura: "Ru underlayer effect on the Fe-Si magnetic film", J. Magn. Soc. Jpn., Vol.29, No.8, pp.826-830 (2005). (in Japanese)

[10] M. Sonehara, T. Sugiyama, T. Ishikawa, K. Inagaki, T. Sato, K. Yamasawa, and Y. Miura, "Relation Between Microwave Complex Permeability and Ferromagnetic Fe-Si Layer Thickness in Mn-Ir/Fe-Si Exchange-Coupled Film,” IEEE Trans. Magn., vol.42, no.10, pp.29842986 (2006). 